\title{
Pyruvate Kinase PKLR Isoform L-Type
}

National Cancer Institute

\section{Source}

National Cancer Institute. Pyruvate Kinase PKLR Isoform L-Type. NCI Thesaurus. Code C157140.

Pyruvate kinase PKLR isoform L-type (543 aa, $~ 58 \mathrm{kDa}$ ) is encoded by the human PKLR gene. This protein is involved in transphosphorylation of phohsphoenolpyruvate into pyruvate and ATP in the liver. 